Results 64 patients participated (median CD4 417/ $\mathrm{mm}^{3}$, range 84-1100). 50 of the patients were treated with single dose BPG. Only one patient had ANS (prevalence 1.56\% 95 CI 0.04-8.4) with CSF RPR negative, CSF TPPA 1:1280 and lymphocytes $45 / \mathrm{mm}^{3}$. Two patients had a pleocytosis (50 and 22 white cells $/ \mathrm{mm}^{3}$ respectively) with negative CSF RPR and CSF TPPA and thus did not meet diagnostic criteria for ANS per protocol.

Discussion/conclusion Our study suggests that single dose BPG is effective treatment for early syphilis in HIV co-infected patients. We will present more data to support this conclusion.

\section{AEROBIC VAGINITIS: PREVALENCE, MANAGEMENT AND OUTCOMES IN A LARGE INTEGRATED SEXUAL HEALTH CLINIC}

${ }^{1}$ Mark Mason*, ${ }^{2}$ Bethan Little, ${ }^{1}$ Andrew Winter. ${ }^{~ S}$ Sandyford Sexual Health Services, NHS Greater Glasgow \& Clyde, Glasgow, UK; ' ${ }^{2}$ niversity of Glasgow School of Medicine, Glasgow, UK

\subsection{6/sextrans-2015-052126.33}

Background/introduction Aerobic vaginitis (AV), a syndrome of abnormal vaginal microflora, was first described in 2002 and is increasingly recognised as a condition distinct from bacterial vaginosis that may require different management.

Aim(s)/objectives To describe the prevalence of moderate-tosevere AV, its management and outcomes in a UK setting.

Methods We included all women presenting to our large integrated sexual health service who met criteria for gynaecological examination and near-patient microscopy. A single biomedical scientist scored the wet mount according to the method of Donders et al. If the score was 5 or above (indicating moderate to severe $\mathrm{AV}$ ) the requesting clinician was informed. We reviewed case notes to determine treatment choice and outcome. Results From 1/12/13 to 30/11/14, 1616 wet films were read. Overall, 314 (19.4\%) had an abnormal AV score (11 (0.7\%) severe AV (score >6), $61(3.8 \%)$ moderate AV (score = 5-6), $253(15.7 \%)$ slight AV (score = 3-4)). Patients with severe AV were significantly older than those with moderate AV (mean age 42.7 vs 32.0 years, $\mathrm{p}=0.04)$, but only $6(8.3 \%)$ patients had atrophic change. Among patients with AV scores of 5 or more, trichomonas was seen in $2(2.8 \%)$ patients, $13(18.5 \%)$ had evidence of yeast infection. First-line treatment included intravaginal clindamycin (49.7\%), oral metronidazole (27.3\%), antifungals, penicillins, acidification gel and local oestrogen. Symptoms persisted in $19.4 \%$, re-occured in $4.2 \%$ and resolved in $43 \%$, with $33 \%$ not re-attending.

Discussion/conclusion Patients with moderate-to-severe AV scores are challenging to manage with a high proportion of repeat attendance. Severe AV occurs in an older population.

\section{Clinical Case Studies: $2^{\text {nd }}$ June 2015}

\begin{tabular}{l}
\hline C1 CASE SERIES: MANAGING DESQUAMATIVE \\
INFLAMMATORY VAGINITIS IN TRANS-MEN
\end{tabular}

Kate Nambiar*, Deborah Williams, Tamara Woodroffe, Alison Parnell, Daniel Richardson. Brighton and Sussex University Hospitals NHS Trust, Brighton, UK

10.1136/sextrans-2015-052126.34
Background/introduction Desquamative inflammatory vaginitis (DIV) is an uncommon condition characterised by florid vaginal inflammation causing vaginal discharge, vulval pain and dyspareunia. Microscopy typically shows absent vaginal flora, numerous polymorphs and immature parabasal cells with no mature epithelia. The pathogenesis of DIV is currently unknown but may involve tissue kallikrein-related peptidases which are regulated by sex hormones and corticosteroids.

Case-1: 35 -year-old trans-man on testosterone for 18-months presenting with yellow vaginal discharge, vestibular pain and dyspareunia. Examination revealed vaginal inflammation and mucopurulent discharge. Microscopy was typical of DIV. He was treated with intravaginal clindamycin reporting a good response.

Case-2: 26-year-old trans-man on testosterone for 7-years presenting with vaginal discharge, dyspareunia and post-coital bleeding. Examination revealed inflamed friable vaginal mucosa. Microscopy findings were typical of DIV and he started treatment with intravaginal clindamycin (partial-response) and switched to intravaginal prednisolone.

Case 3: 20-year-old trans-man with vaginal discharge and post-coital bleeding who started testosterone 6-months earlier. Examination and microscopy findings were typical of DIV. He commenced treatment with intravaginal clindamycin (partialresponse) and switched to intravaginal prednisolone.

Case 4: 19-year-old trans-man on testosterone for 9-months presenting with vaginal pain and bleeding. Examination and microscopy were typical of DIV. He started treatment with intravaginal clindamycin (partial-response) and switched to intravaginal prednisolone.

Discussion We present four cases of DIV in trans-men possibly associated with androgens responding to intravaginal clindamycin and steroids. As well as causing significant morbidity DIV may increase transmission of sexually-transmitted-infections in trans-men: we need to understand more about its aetiology, management and long term outcomes.

\section{C2 GONOCOCCAL TENOSYNOVITIS IN TWO HIV-INFECTED HETEROSEXUAL MALES: DELAYED DIAGNOSES FOLLOWING NEGATIVE URINE NAAT TESTING}

Jonathan Shaw*, Peter Flegg, John Sweeney. Blackpool Sexual Health Services, Blackpool, UK

\subsection{6/sextrans-2015-052126.35}

Background Disproportionately high gonococcal incidence rates amongst men have altered the clinical picture of disseminated gonococcal infection (DGI). The 'classical' female patient experiencing a triad of arthritis, tenosynovitis and cutaneous lesions no longer predominates. We present two cases emphasising the need for thorough investigation with evident clinical signs of DGI.

Cases A 48 year old Nigerian heterosexual male presented with a $6 \mathrm{~cm}$ inguinal mass and oral hairy leukoplakia. Impression was of lymph node abscess; HIV testing was positive. Urine Nucleic Acid Amplification Testing (NAAT) for chlamydia and gonorrhoea (CT/GC) was negative. Subsequently he developed a swollen tender left wrist. Inguinal abscess aspiration for NAAT testing returned a positive gonococcal result. Treatment was instigated with intravenous ceftriaxone for 4 days, subsequently switching to cefixime for a further week. 3 weeks later his wrist swelling resolved. 
A 50 year old HIV-positive British heterosexual male presented after returning from Thailand. He had developed a tender swollen left wrist. Urine NAAT for CT/GC was negative. He reported condomless oral and vaginal sex with multiple Thai females. Gonococcal tenosynovitis was suspected and extragenital NAATs and cultures for CT/GC were taken; NAAT for pharyngeal gonorrhoea was positive. Single dose ceftriaxone and azithromycin was prescribed, followed by cefixime for 1 week. Two weeks later his symptoms cleared.

Conclusion Reflecting on these cases a DGI diagnosis was attained following careful consideration of possible differentials and persistence in identifying Neisseria gonorrhoeae. Both diagnoses would have been missed if following current testing guidance which recommends penile-only sampling of heterosexual men.

\section{C3 SYPHILITIC AORTITIS IDENTIFIED IN A PATIENT NEWLY DIAGNOSED WITH HIV - THE EMERGING TIP OF AN ONCOMING ICEBERG?}

${ }^{1}$ Noel B Connolly*, ${ }^{1}$ Jonathan Shaw, ${ }^{2}$ Cara Hendry, ${ }^{1}$ Margaret Kingston. ${ }^{1}$ Manchester Centre for Sexual Health, The Hathersage Centre, Manchester Royal Infirmary, 280 Upper Brook Street, Manchester, UK; ${ }^{2}$ Manchester Heart Centre, Manchester Royal Infirmary, Oxford Road, Manchester, UK

\subsection{6/sextrans-2015-052126.36}

Background A 38 year old man presented for HIV testing following his male partner's diagnosis. Examination revealed systolic and decrescendo diastolic heart murmurs, palpable thrill, bounding pulses, and positive Corrigan's sign. He had not tested previously for HIV or syphilis and had been in a monogamous relationship for 8 years. We describe this man who was asymptomatic - from both HIV and aortic valve disease - with incidental diagnosis of severe syphilitic aortitis following partner notification for HIV.

Results HIV antibody test was positive with baseline viral load 239505 copies/ml and CD4 count 103 cell $/ \mu \mathrm{L}$ (8\%). Syphilis serology was positive with rapid plasma reagin (RPR) 1:4. CXR was unremarkable. ECG was consistent with left ventricular hypertrophy with strain. Echo revealed severe mixed aortic valve disease, left ventricular hypertrophy, good LV systolic function and normal aortic arch appearance. He commenced prednisolone $60 \mathrm{mg}$ OD for $5 \mathrm{~d}, 72 \mathrm{hr}$ before starting three weekly doses of 2.4 MU benzathine penicillin. He was admitted for $48 \mathrm{hr}$ for cardiac monitoring at the start of treatment - which proceeded with no complication. Multidisciplinary involvement with GU physicians, cardiologists and cardiothoracic surgeons was instigated from the start with aortic valve \pm root replacement planned imminently.

Discussion Resurgence of syphilis in the UK was reported in the late 1990s with an ongoing epidemic since, mainly involving MSM. Cardiovascular syphilis typically occurs 15-30 years following primary infection with Treponema pallidum, with complications in $10 \%$ of cases. Could this man be amongst the first cases to develop tertiary syphilis in this latest epidemic?

\section{C4 A COMPLICATED CASE OF CANDIDA}

Fiona Cresswell, Catherine Kirby*, Deborah Williams. Brighton and Sussex University Hospital, Brighton, UK

10.1136/sextrans-2015-052126.37
Background Vulvovaginal candidiasis (VVC) is a common condition caused by Candida albicans in 80-92\%. Candida robusta is rarely identified in humans and has only been reported as a cause of VVC in pregnant women. We present a case of chronic Candida robusta VVC.

Case A 25 year-old, on Cerazette, presented to her GP with discharge and vulval itching; treatment with clotimazole was effective but symptoms recurred. In clinic, one month later, a clinical and microscopic diagnosis of VVC was made, she was treated with fluconazole plus econazole pessary and cream. HIV, syphillis, gonorrhoea and chlamydia were negative.

Despite initial improvement she represented with recurrent symptoms, microscopy and culture again confirmed Candida species. Following a fourth presentation oral fluconazole $150 \mathrm{mg}$ every $72 \mathrm{~h} \times 3$ followed by a weekly dose for three months was commenced. She was asymptomatic during this time but relapsed on discontinuation. Microscopy again confirmed spores and on speciation Candida robusta sensitive to fluconazole was isolated. A second 3-month fluconazole course was given. She had now developed provoked vulvodynia. Low-grade symptoms persisted and Candida robusta was again cultured, now resistant to fluconazole. A one-week course of oral voriconazole was given. Follow-up microscopy was negative but her vulvodynia had worsened. Treatment with amitriptyline was commenced and on review two months later culture remained negative and her vulvodynia had improved.

Discussion We report a case of chronic Candida robusta VVC in a non-pregnant immunocompetant woman, which acquired fluconazole resistance and precipitated vulvodynia. Speciation and sensitivity testing are important in women with recurrent symptoms.

\section{C5 A CASE OF REPEATED RHABDOMYOLYSIS ASSOCIATED WITH PEPSE: AN UNCOMMON SIDE EFFECT OF RALTEGRAVIR}

Jennifer Murira*, Sarah Schoeman. The Leeds Centre for Sexual Health, Leeds, UK

\subsection{6/sextrans-2015-052126.38}

Background/introduction The first line regime for PEPSE recently changed to Truvada/Raltegravir. We report on a case of rhabdomyolysis associated with Raltegravir.

Case A 25 year old MSM requested PEPSE in February 2013. Commencing Truvada/Kaletra, he switched to Truvada/Darunavir/Ritonavir due to arthralgia. He further received Truvada/Darunavir/Ritonavir 4 months later for another PEPSE request.

A third PEPSE episode was initiated in September 2014 commencing Truvada/Raltegravir. Baseline investigations showed an eGFR $75 \mathrm{ml} / \mathrm{min} / 1.73 \mathrm{~m}^{2}$. Two weeks later the patient was complaining of severe myalgia/lethargy. Also he noticed his urine colour change to brown. Repeat investigations were: creatinine $121 \mathrm{umol} / \mathrm{L}$, eGFR $62 \mathrm{ml} / \mathrm{min} / 1.73 \mathrm{~m}^{2}$, Creatine Kinase (CK) $1392 \mathrm{iu} / \mathrm{L}$, urine protein/creatinine (uPCR) $2.9 \mathrm{mg} / \mathrm{mmol}$. On urgent review he was admitted for IV rehydration and cessation of PEPSE having developed an acute kidney injury and rhabdomyolysis. His CK fell following fluid replacement.

In November our patient was seen again having self-initiated PEP following a needle-stick injury from a used needle. He had taken 1 Truvada/Raltegravir from left over medication. However he had recurring myalgia and lethargy. His repeat CK was 2625 $\mathrm{iu} / \mathrm{L}$. The regime was immediately stopped, however his muscle pains and weakness continued for 3 weeks with a slow decline in his CK. 